\title{
The Study of Vehicle Bumper Crash Characteristics
}

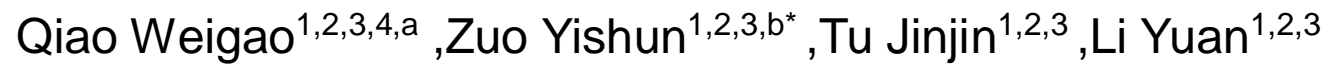

1. Hubei Collaborative Innovation Center for Automotive Components Technology, China;

2. Hubei Key Laboratory of Advanced Technology for Automotive Components, China;

3.Wuhan University of Technology, School of Automotive Engineering, wuhan,China; 4.Wuhan University of Technology, Huaxia College,Wuhan,430223, China

awgqiao@126.com, ${ }^{\mathrm{b} 1326403165 @ q q . c o m, ~ * C o r r e s p o n d i n g ~ a u t h o r ~}$

Keywords: Car bumper, collision, explicit nonlinear finite element, LS-DYNA.

\begin{abstract}
With the mass production of cars and utilities, the incidence of car accidents is increasing, while the case of car accidents in a variety, collision probability is highest. The theory of the explicit nonlinear finite element which is adopted in the crash simulation is expounded. According to the European regulation of ECE-R42, a finite element model of low-speed crash between car bumper system and pendulum are established. The stress and strain contours, the displacement of node which on the deformation parts of bumper beam, the amount of compression of crash box and the process of energy conversion, etc. In the simulation results are objectively and deeply analyzed through HyperView. Finally, the paper discusses the influence factors on collision characteristics, such as the section shape, thickness and material properties of bumper beam.
\end{abstract}

\section{Introduction}

In recent years, with the rapid development of economy in our country , the amount of car increases quickly, the speed of car becomes more and more fast which causes the increasing incidence of car accidents. While the case of car accidents in a variety, collision probability is highest. Car crash means the car experiences an intense collision which occurs in a very short time, it is a complex physical process of transient that contains the structure's geometric non-linearity shown by large displacement, large rotation and large strain and material's physics non-linearity. Bumper plays an important role in load bearing and energy absorption when vehicle experiences frontal impact. Therefore, studying the bumper's collision characteristics and improving the bumper's ability of energy absorption have a vital significance to protect people's security.

\section{The calculation method of bumper crash simulation}

Because of car collision's instantaneity and large deformation of structure, materials not only have a certain strain rate, but also stays in the elastic-plastic state, but there is no one-to-one relationship between stress and strain once the material transforms into the plastic state when it's beyond the yield limit. It means that strain not only depends on the stress state but also depends on the whole loading process. Therefore, small integration time step must be used in the calculation in order not to damage the material constitutive relation. So the car crash simulation process uses the explicit nonlinear finite element method. When we adopt the non-linear dynamic finite element method to deal with large-scale engineering problems, its fatal disadvantage is that it costs too much time. The data storage and operation times can be greatly saved when we use single-point gauss integral element, but a single point of integral may cause zero energy mode(Hourglass mode)which will cause serious distortion calculation results that makes it more difficult to solve the equation or even fail. Therefore, the hourglass mode should be controlled by 
human.

CATIA、HyperMesh and LS- DYNA are used in this simulation in order to get the more accurate results. The geometric model was set up by CATIA and pre-processed through HyperMesh, then the key files are generated and submitted to LS-DYNA for numerical simulation. After simulation, HyperView post-processing program is used to analyze the simulation results.

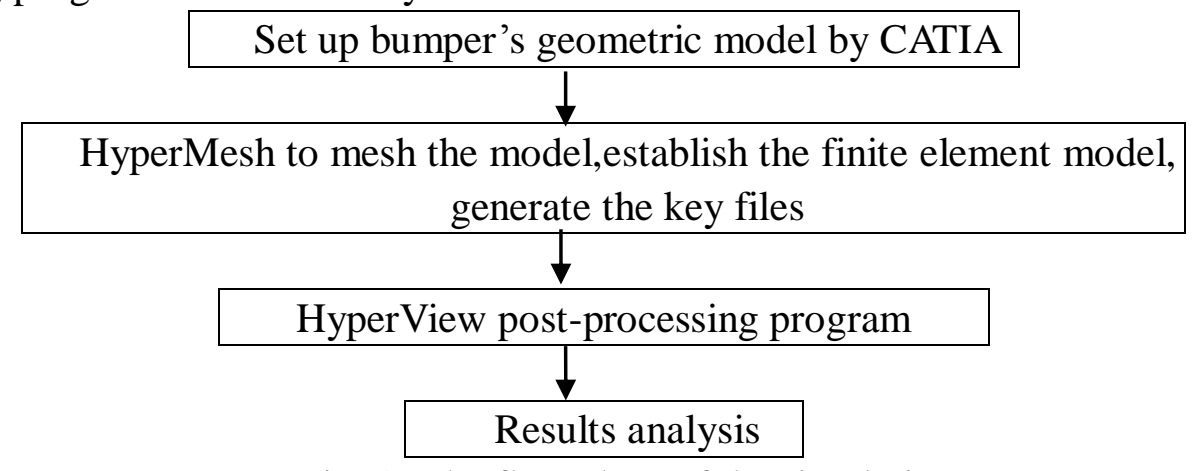

Fig. 1: The flow chart of the simulation

\section{2 . The establishment of the model}

\subsection{The establishment of the bumper model}

Bumper model consists of two parts: one is the bumper beam, the other is a suction box. Two parts are connected by welding. We use CATIA to set up bumper collision system entity model based on measured parameters of a car bumper. The pendulum's three-dimensional model is established according to ECE-R42 regulations.

Considering that the actual structure of the original bumper is very complicated, so the model was simplified. Different from static finite element analysis, impact analysis is mainly focused on calculating structure deformation, so more consideration should be taken into deformation parts when modeling. For car collision, thin-walled metal, which can change dramatically and its shape is complex and sensitive to external load and boundary conditions, is the most important parts of absorbing deformation energy. Under normal circumstances, the tiny hole structure and the connection parts can be simplified or even ignored when modeling. The car body parts behind the longitudinal beams are simplified by a plane with mass.

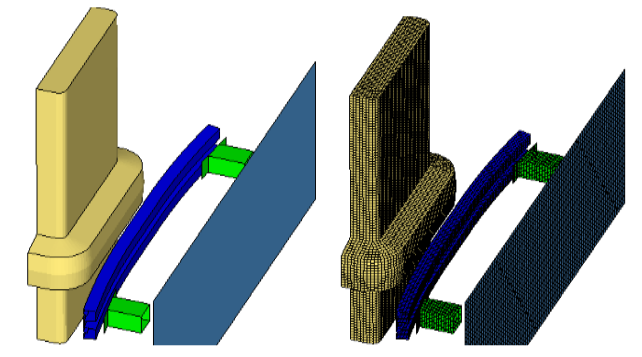

Fig.2: Three dimensional geometry model and finite element model of the bumper and pendulum

Due to the integral loss of BT shell element is minimum, it suggests that the computer calculation speed is very fast, so it has become an indispensable shell element formula. Under most circumstances, it is the most stable and effective formula in dealing with large deformation problems, so we adopt BT shell element. The cell size we selected is $10 \mathrm{~mm}$. Finally, we position each part according to the requirements of the collision. The quality of the grid cell should be checked and evaluated before simulating, high quality of the unit is the primary factor ensuring the calculation precision and calculation efficiency. If the quality of grid cell is poor, it may sometimes lead to the non-convergent calculation results and greatly decrease the calculation accuracy when explicit algorithm was selected. The simulation results will have no practical guidance value. 


\subsection{Material and properties setting}

Most of materials used in car body are steel. The bumper system plays an important role in load transmission and energy absorption in the frontal crash at low speed. In recent years, in order to realize auto lightweight and achieve the effect of energy conservation and emissions reduction, aluminum is more and more widely used in automobile materials.

In a car crash or covering parts stamping analysis, MAT24 material is mainly used for some isotropic materials, when using this model, these parameters should be input: the elastic modulus, density, poisson's ratio, the equivalent plastic strain of material failure, strain rate parameters $\mathrm{C}$ and $\mathrm{P}$, piecewise function which shows the stress and strain of materials etc.

MAT20 material is rigid material model, so the deformation of parts using MAT20 is not considered. All the nodes will keep the relative position unchanged, degrees of freedom are coupled to the mass center of the rigid body, rigid body only has six degrees of freedom regardless of the amount of nodes that the finite element model defines. Therefore, it can greatly reduce the CPU calculation time, the mass, center of mass and inertia properties are automatically calculated by computer through the volume and density of each unit .Force and displacement acting on the rigid body is obtained by the superposition of node values of each time step and the corresponding displacement values are passed to the node, Users need to input the actual material density, elastic modulus and poisson's ratio. Basic parameter Settings are as follows.

Table 1: The material and properties of each part

\begin{tabular}{cccccc}
\hline $\begin{array}{c}\text { The type of } \\
\text { material }\end{array}$ & $\begin{array}{c}\text { Density } \\
(\rho) \mathrm{kg} / \mathrm{m}^{3}\end{array}$ & $\begin{array}{c}\text { the elastic } \\
\text { modulus(E)MPa }\end{array}$ & $\begin{array}{c}\text { poisson's } \\
\text { ratio }\end{array}$ & $\begin{array}{c}\text { yield } \\
\text { strength }\end{array}$ & part \\
\hline MAT24 & 7850 & 215000 & 0.3 & 800 & beam \\
MAT20 & 7850 & 215000 & 0.3 & 270 & $\begin{array}{c}\text { suction box } \\
\text { pendulum }\end{array}$ \\
\hline
\end{tabular}

The thickness of the bumper beam and energy absorption box are set to $1.29 \mathrm{~mm}$. The energy absorption box is made from low carbon steel which has relatively low yield limit and is prone to yield deformation. Low carbon steel can quickly enter the stage of yield which absorbs impact kinetic energy in the collision process.

\subsection{Connection Settings}

Bumper beams and suction box are deformable parts, we can establish the corresponding solder joints on the two nodes to connect them. The welding units used in this welding method do not need materials and properties, Fault will happen in the calculation if we set its materials and properties. Connection between suction box and car body adopts the common nodes. Because in this paper body parts is simplified to a plane with mass which does not consider the deformation, connection between deformable parts and non-deformable parts cannot use welding method. Hence, the common nodes are used.

\section{The results analysis of impact simulation}

According to the test standard, the effective mass of pendulum should be equal to the weight of vehicle. According to the parameter of the selected models, it is $1220 \mathrm{~kg}$. Pendulum falls freely from a certain height (406.4-508mm)and impact bumpers with speed of $4 \mathrm{~km} / \mathrm{h}$ which belongs to low-speed collision. The dynamic response characteristics of the bumper in a low-speed collision refers to the time response of the process of the bumper's displacement (or deformation), velocity, acceleration and so on. The analysis of all kinds of time response process plays an important role in studying the collision features of the 
bumper.

Setting the computation time of the collision to $100 \mathrm{~ms}$, we intercept the bumper's stress and strain contours when the time is $0 \mathrm{~ms}, 15 \mathrm{~ms}, 30 \mathrm{~ms}, 50 \mathrm{~ms}, 60 \mathrm{~m}$ and $80 \mathrm{~ms}$. As shown in figure 3.

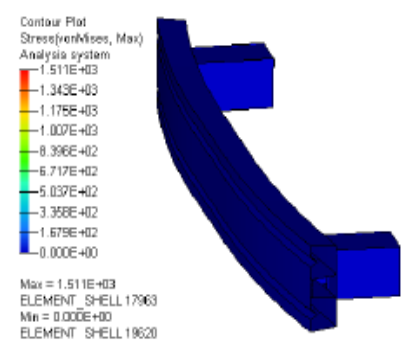

a) $\mathrm{t}=0 \mathrm{~ms}$

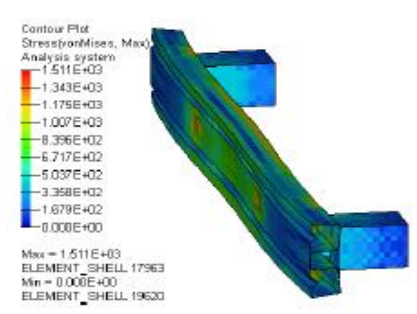

d) $\mathrm{t}=50 \mathrm{~ms}$

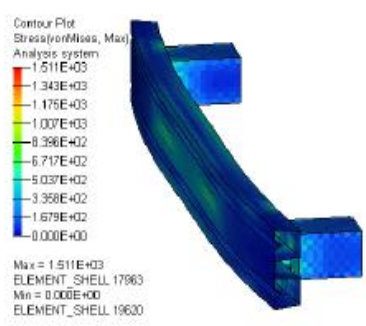

b) $\mathrm{t}=15 \mathrm{~ms}$

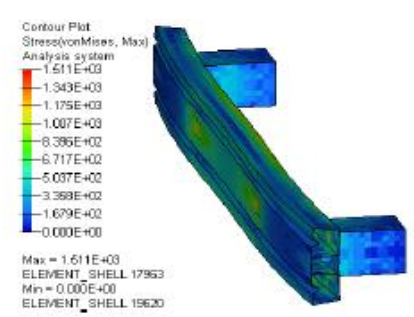

e) $\mathrm{t}=60 \mathrm{~ms}$

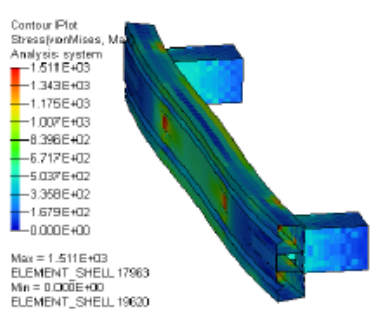

c) $\mathrm{t}=30 \mathrm{~ms}$

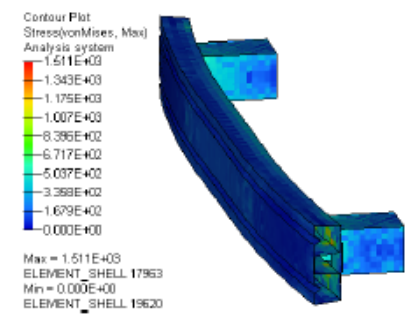

f) $\mathrm{t}=80 \mathrm{~ms}$

Fig. 3: Bumper stress and strain contours at each time

By observing the stress and strain of the bumper system, we can find that the front of the bumper beams has slight deformation which means that the collision has occurred at $t=15 \mathrm{~ms}$. Then the relative displacement of the beam and the pendulum continues to grow, at about $t=50 \mathrm{~ms}$ the plastic deformation reaches the maximum. The deformation tends to decrease. We can see that most of the stress and strain contours is blue at $\mathrm{t}=80 \mathrm{~ms}$, the stress is only concentrated in the place where the bumper beam and the suction box contacts, other parts basically recovers to the initial state. According to the above analysis, we can conclude that $30-50 \mathrm{~ms}$ is the time interval when elastic-plastic deformation of beam reaches the maximum. The system energy form changes dramatically at this time interval, the kinetic energy of the pendulum is converted into internal energy of the bumper system which means impact energy is absorbed. The maximum stress of the beam in the process of collision is $1511 \mathrm{Mpa}$, the yield stress value of the material we use is $800 \mathrm{Mpa}$, therefore, it has obvious plastic deformation. Because it is a low-speed collision, initial velocity is small, the pendulum begins to show springback after 50ms, the deformation of the bumper gradually restores in time interval of 50-100ms.Considering the rigid pendulum features makes it not happen any elastic-plastic deformation, so we hide the pendulum in the figure. We, however, can still clearly see the inward bending deformation on the center of the bumper beam after the collision which is one of the most serious stress concentration place.

\section{Structure improvement of bumper system}

Optimization of bumper structural mostly focused on the following aspects, length, thickness, cross-sectional shape of the bumper beams. From the shape and energy-absorbing crumple box mechanism to improve aspects of design optimization, from the energy-absorbing cartridge slot induction location shape dimensions to improve the design optimization and use new materials. The research of new materials, new technology aimed at ensuring the realization of the bumper crash worthiness premise lightweight design.

This paper attempts to improve the original bumper's cross-sectional shape, thickness, material and so on, in order to get better energy absorption effect. 


\subsection{The Impact of the cross-sectional shape}

Change the original "B" shaped cross-section into "Lu" shape, but don't change the width, thickness and other parameters. When designing the "Lu" cross-section shape, we should keep constant cross-sectional area. Collision simulated stress strain contours shown in Figure 4.
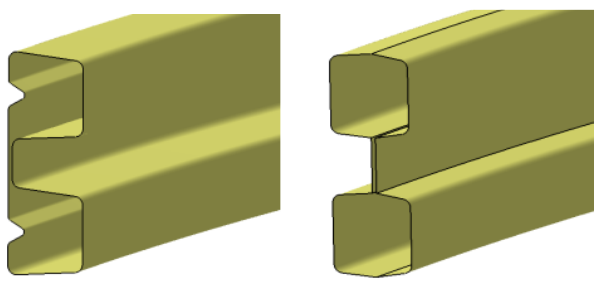

Fig. 4: Structure of the original bumper beam (left) and an improved structure (right)

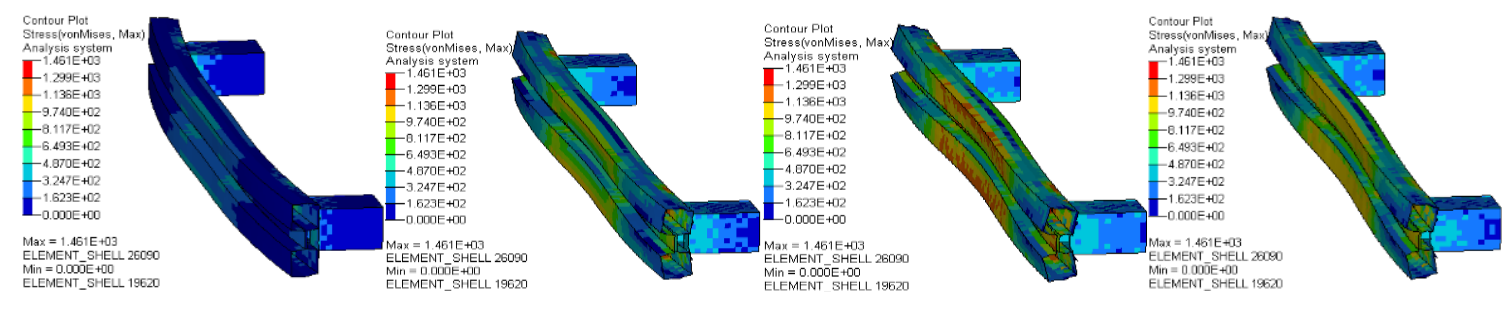
a) $\mathrm{t}=15 \mathrm{~ms}$
b) $\mathrm{t}=30 \mathrm{~ms}$
c) $\mathrm{t}=50 \mathrm{~ms}$
d) $\mathrm{t}=60 \mathrm{~ms}$

Fig.5: Bumper stress and strain contours at each time

Watching the simulated result of the "Lu" shaped cross-section of the bumper, we can obviously find that in the front of the beam has a slight deformation at $15 \mathrm{~ms} .30 \mathrm{~ms}-50 \mathrm{~ms}$ is the time when the beam has a greater elastic-plastic deformation, and then the pendulum begin to rebound, the beams and energy-absorbing box deformation gradually restored. The maximum stress of the entire collision process is $1461 \mathrm{MPa}$.It is over the yield stress limit of this material which is $800 \mathrm{MPa}$. But comparing the maximum stress value of the original structure of $1511 \mathrm{MPa}$, it has reduced.

\subsection{The Impact of the Beam Thickness}

The thickness of the original bumper beam is $1.29 \mathrm{~mm}$, due to the convenience of computer simulation, the thickness values can be modified to observe its influence to collision performance. We set its thickness to $1 \mathrm{~mm}$ and $1.8 \mathrm{~mm}$, the stress-strain contour of the collision process (here only the interception of the $\mathrm{t}=50 \mathrm{~ms}$ and $\mathrm{t}=80 \mathrm{~ms}$ ) are shown in Figure 6 .

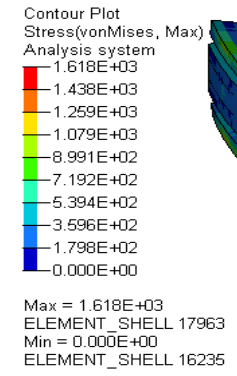

a) $\mathrm{t}=50 \mathrm{~ms}$

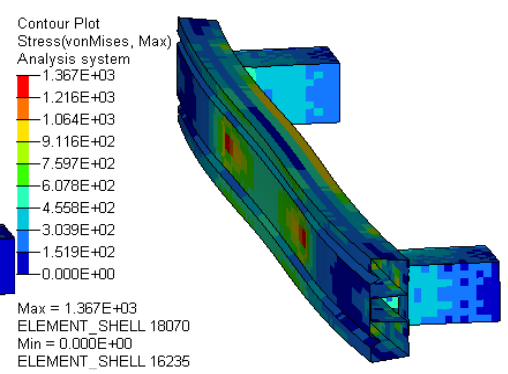

b) $\mathrm{t}=80 \mathrm{~ms}$

Fig.6: Bumper stress and strain contours at each time

As is shown in the figure that $1 \mathrm{~mm}$ beam deformation is greater than $1.8 \mathrm{~mm}$ beam, but it doesn't not exceed the distance between the beams and the body which has met the crash worthiness requirements. Their maximum stress in the collision process is $1618 \mathrm{MPa}$ and $1367 \mathrm{MPa}$, which beyond the yield limit of the beam, its plastic deformation occurs. The result is very realistic, relatively thin-walled beam intensity "soft", large deformation, but we also need to continue to compare their energy absorption characteristics. 


\subsection{The Impact of the Beam Material}

In contrast to the above analysis, we found that the safety performance and the lightweight of the car can coexist. There are two ways to achieve the lightweight of bumper. Firstly, improve the structure, this paper has no effective optimization, so the improved energy-absorbing bumper effect is not obvious. The second is the use of low-density high-strength lightweight materials. Such materials are:

1) A low density material, such as aluminum alloy, composite materials etc.

2) High-strength materials, such as high strength steel.

In this paper, we use the aluminum alloy material which is more and more widely used in vehicle body. The collision process simulated stress strain contours shown in figure 7.

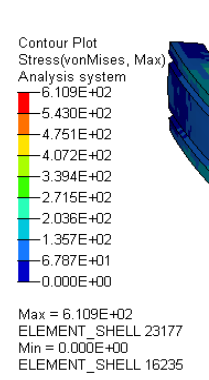

a) $\mathrm{t}=30 \mathrm{~ms}$

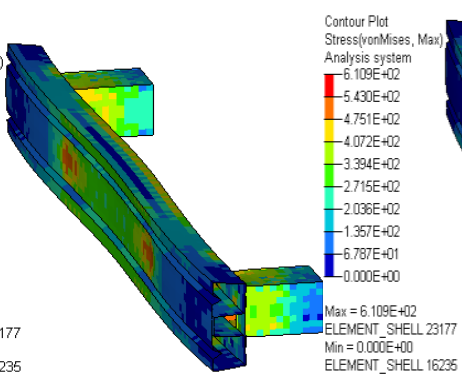

b) $\mathrm{t}=50 \mathrm{~ms}$

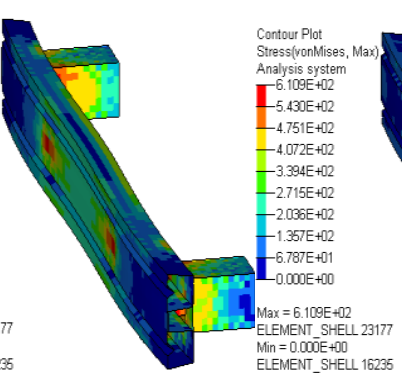

c) $\mathrm{t}=60 \mathrm{~ms}$
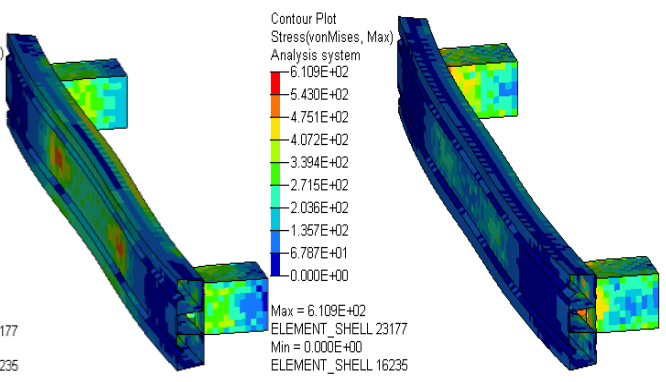

d) $\mathrm{t}=80 \mathrm{~ms}$

Fig.7: Bumper stress and strain contours at each time

The setting of the material properties does not affect the starting time of the collision. The collision starts at $\mathrm{t}=5 \mathrm{~ms}$ and the front of the beam has a slight deformation at $15 \mathrm{~ms}$.During $30 \mathrm{~ms}-50 \mathrm{~ms}$, it experiences a greater elastic-plastic deformation. Then the pendulum begins to rebound, the deformation of the beams and energy-absorbing box gradually restore. The maximum stress of the entire collision process is $610.9 \mathrm{MPa}$ and the yield stress limit of the used material $7075-\mathrm{T} 6$ aluminum alloy is $524 \mathrm{MPa}$, a little plastic deformation occurs. The yield stress of the original bumper beam structure is $800 \mathrm{MPa}$, and the maximum stress of the collision process is $1511 \mathrm{MPa}$, the plastic deformation is more serious.

\section{Conclusions}

From the study of this paper, we found that there are little impacts of the cross-sectional shape on the crash performance, the original energy value of per unit mass absorbed can be increased when we reduce the thickness of the beam to some extent, so it is not necessary to increase the thickness of the beam. The application of aluminum alloy not only reduces the weight of bumper but also reinforces the crash performance of bumper. In this paper, by studying the characteristics of low speed impact bumper, it provides some guidance on in-depth study of a frontal collision car and it has a certain reference value for the design and development of bumper beams.

\section{References}

[1]Hu Yuanzhi, Zen Biqiang, Xie Shugang. Simulation and Analysis of Vehicle Safety Based on LS-DYNA[M]. Beijing: Tsinghua University Press, 2011,9.

[2]Sun Xiaoqiang. A study on the Crashworthiness of Passenger Car in Low-speed Front Impact [D], Changsha: Hunan University.

[3]Zhang Jing, Zhang Ruiqian. Finite Element Analysis of Vehicle Bumper Collision [J], Theory of Urban Construction, 2011(35).

[4]Bai Jingze, Theoretical Foundation and Case Study Of LS-DYNA3D [M].Beijing: Science Press, 2005.

[5]Wei Longkun, Yang Rongsong, ZhangYong. Finite element simulation and optimization of car bumper collision [J] Modern Manufacturing Technology and Equipment,2008(3). 
